WTEREFACE TEENOLÓGCACA

\title{
APLICABILIDADE DOS CONCEITOS DE GESTÃO DO CICLO DE PEDIDO EM UM MODELO DE REVENDA DIRETA DE COSMÉTICOS
}

\author{
APPLICABILITY OF THE ORDER CYCLE MANAGEMENT CONCEPTS IN A \\ DIRECT RESALE MODEL OF COSMETICS
}

\begin{abstract}
Laudiceia Gonçalves de Aguiar - laudicei@hotmail.com
Rhadler Herculani - rhadler.herculani@fateccbb.edu.br

Faculdade de Tecnologia de Bebedouro (Fatec) - Bebedouro - São Paulo - Brasil
\end{abstract}

DOI: 10.31510/infa.v17i1.730

\begin{abstract}
RESUMO
O objetivo desse artigo foi compreender a aplicabilidade do ciclo de pedido de revenda direta em uma empresa do ramo de cosméticos, identificando seus agentes, e sugerir a aplicação de conceitos de gerenciamento de nível de serviço e gestão logística. Entende-se que o sucesso do gerenciamento da cadeia de suprimentos está atrelado a integração de todos os processos referentes as atividades que envolvem a produção, circulação e destruição de produtos e serviços entre as empresas, seus fornecedores e clientes. Por isso, a abordagem do artigo se justifica pela proximidade do modelo de negócio de venda direta no dia-a-dia de muitas pessoas na nossa região, e o fato de que identificar e aplicar conceitos logísticos pode proporcionar benefícios para o desenvolvimento econômico da sociedade. Para isso, utilizou-se como metodologia de estudo uma pesquisa bibliográfica de caráter qualitativo e de um estudo de caso . Os resultados demonstraram que o problema da variabilidade de tempo está relacionado principalmente ao fato da informalidade e da simplicidade desse tipo de comercialização de produtos, na maior parte dos casos, não são usadas ferramentas para padronização e controle. Assim, conclui-se que é possível aplicar conceitos de gerenciamento logísticos a pequenos negócios, mesmo que informais e pouco estruturados.
\end{abstract}

Palavras-chave: Venda direta. Cadeia de Suprimentos. Nível de Serviço.

\begin{abstract}
The objective of this article is to understand the applicability of the direct resale order cycle in a cosmetics company, identifying its agents, and to suggest the application of service level management and logistics management concepts. It is understood that the success of supply chain management is linked to the integration of all processes related to the activities that involve the production, circulation and destruction of products and services between companies, their suppliers and customers. Therefore, the approach of the article is justified by the proximity of the direct selling business model in the daily lives of many people in our region, and the fact of identifying and applying logistical concepts can provide benefits for the economic development of society a return. For this, a qualitative bibliographic research and a real case study were used. The results showed that the problem of time variability is mainly
\end{abstract}


related to the fact that the informality and simplicity of this type of product commercialization, in most cases, tools for standardization and control are not used. Thus, it is concluded that it is possible to apply logistic management concepts to small businesses, even if informal and poorly structured.

Keywords: Direct sale. Supply chain. Service level.

\section{INTRODUÇÃO}

Dentre os diversos fatores que impulsionaram os avanços na logística mundial, pode-se destacar o aumento exponencial da utilização de ferramentas ligadas a tecnologia da informação, estas que proporcionaram maior flexibilidade e agilidade aos processos logísticos (BALLO, 2006).

Abrangendo todos os agentes integrantes da cadeia produtiva, com benefícios que vão desde a busca por maior qualidade em serviços até minimização dos custos envolvidos, impactando diretamente no nível de serviços oferecidos aos clientes internos e externos (FLEURY; WANKE; FIGUEIREDO, 2000).

Ainda, interpretando as palavras de Fleury, Wenke e Figueiredo (2000), a rapidez no fluxo e a segurança da informação buscam eficiência nos resultados através de minimização de erros, controle efetivo dos custos, isenção da necessidade de retrabalho, focando no atendimento do cliente e posicionamento no mercado.

A visão integrada dos processos logísticos junto a inteligência da informação, adere ao conceito de que uma organização é formada por um sistema integrado por processos e seus subprocessos em busca do objetivo almejado (MAXIMIANO, 2008). O processamento de pedido e a análise do ciclo de pedido fazem parte fundamental do processo logístico com foco no atendimento ao cliente. Analisar o desempenho das atividades envolvidas, seja com o uso de indicadores ou controles personalizados, possibilita o monitoramento da performace da empresa ou dos enredados dentro do sistema (NOVAES, 2007).

O processamento de pedidos dentro da empresa merece atenção especial dos gestores e operadores, por tratar-se de uma atividade primária dentro do sistema logístico e o apropriado gerenciamento dos seus processos é fundamental para garantir o nível de serviço ansiado pelos clientes (BALLOU, 2006).

Assim, este artigo busca investigar através de estudos os temas nível de serviço ao cliente e o atendimento do ciclo de pedido com eficiência e efetividade, auxiliando no 


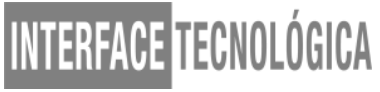

desenvolvimento estratégico da logística de distribuição. Através de leituras e levantamentos bibliográficos de autores diversos, buscou-se resposta para sobre se os conceitos de ciclo de pedidos são aplicáveis a pequenos negócios de revenda direta de cosméticos baseado em um estudo de caso.

Segundo levantamento da Associação Brasileira de Vendas Diretas (ABEVD, 2018), disponível no site oficial, o setor de venda direta no Brasil movimentou R \$ 45 bilhões em 2018 e envolveu mais de 4 milhões de empreendedoras independentes, que atuaram nos ramos de: saúde, beleza, utilidades domésticas entre muitos outros.

O objetivo desse estudo foi compreender a aplicabilidade dos conceitos de ciclo de pedido para o modelo de revenda direta de produtos cosméticos de grandes empresas, identificando seus agentes e sugerindo a aplicação de conceitos de gerenciamento do nível de serviço e gestão logística.

\section{REVISÃO DA LITERATURA}

\subsection{Principais aspectos da logística de distribuição}

O sucesso do gerenciamento da cadeia de suprimentos está atrelado a integração de todos os processos referentes as atividades que envolvem a produção, circulação e destruição de produtos e serviços entre as empresas, seus fornecedores e clientes. E para garantir o nível de serviço deve-se atender as expectativas relacionadas a velocidade e confiabilidade na disponibilização dos mesmos (BALLOU, 2006).

Bowersox (1996), relaciona a velocidade e precisão do processamento de pedidos ao nível de serviço ao cliente, ou seja, quanto mais tempo o ciclo de pedido levar para ser concluído menor tenderá ser a satisfação do cliente, impactando nos custos e consequentemente no resultado financeiro da empresa. O nível de serviço oferecido na logística pode ser medido com indicadores de desempenho, ferramentas que buscam meios para identificar o quão eficiente estão sendo os resultados dos processos envolvidos diante dos objetivos estabelecidos. 


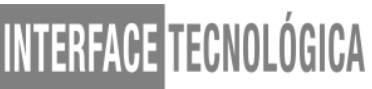

\subsection{Nível de serviço}

Diante a visão estratégica da logística e de forma sistêmica, o serviço ao cliente vai desde o recebimento do pedido, as atividades envolvidas na disponibilização ao cliente final até logística reversa (ou devoluções por quaisquer motivos), além de estar relacionado as áreas comerciais e de marketing, na busca por posicionamento de mercado e vantagens competitivas (BOWERSOX et. al. 2006).

Com outras palavras Novaes (2007) define o nível de serviço ao cliente como todos os processos de forma integral de atendimento do pedido, ou seja, desde a recepção do pedido, a seleção da forma de pagamento e sua efetivação, seleção dos "produtos", disposição para embalagem, preparação para embarque e entrega, serviços aos usuários finais e logística reversa (aceitação a possíveis devoluções).

O propósito da logística é oferecer aos clientes um nível de qualidade que atenda suas expectativas com o objetivo de reduzir custos em toda a cadeia de distribuição. Desenvolver estrategicamente a logística e ter como meta excelência em seus serviços alinhados a custos minimizados é uma excelente ferramenta de diferencial competitivo (CHRISTOPHER, 1999).

Kerenn e Shycon (apud BALLOU, 2006), citam a analogia entre os resultados financeiros (contribuição na lucratividade) esperados e as expectativas do cliente: clientes satisfeitos - quando a empresa atinge o nível de serviço estabelecido de forma satisfatória garantem aumento na participação no mercado, destaque entre concorrentes e consecutivamente crescente lucratividade.

\subsection{Ciclo de pedido}

De forma simplificada pode-se entender o ciclo de pedido como o tempo e as atividades necessárias para ligar o "fornecedor" ao "cliente” dentro de uma operação logística.

Para Bae (2012), criar fatores que compõem a integração logística trabalham em prol da criação de valor para o cliente, e tratam o tempo do ciclo de pedido como elemento crítico na gestão logística. O conceito de "tempo" leva a outra linha de raciocínio na disponibilização de produtos que é a localização das instalações, os transportes necessários, a real necessidade de estoque, armazenagem e manuseio dos produtos assim como as embalagens.

Nas palavras de Santo (2003, p. 102): 
[...] o ciclo do pedido do cliente inclui o tempo que se passa entre a colocação do pedido até o seu recebimento no estoque do cliente, consistindo, tipicamente, das seguintes etapas: (1) preparação e transmissão do pedido; (2) recebimento e entrada do pedido; (3) processamento do pedido; (4) resgate no estoque e embalagem; (5) expedição do pedido e (6) entrega e descarregamento no cliente.

Os elementos do ciclo de pedido segundo Motta e Bianchini (2006), podem ser divididos em: tempo de transmissão e processamento do pedido, tempo de montagem do pedido, a disponibilidade de estoque, o tempo para produção e já relacionado ao transporte de forma mais direta, a entrega final. Lalonde e Zinzer (1976) acrescentam a esse conceito o elemento de análise de crédito e validação das informações cadastrais, fatores que podem atrasar ou interromper o atendimento ao pedido.

Figura 1 - Representação dos subprocessos do ciclo de pedido

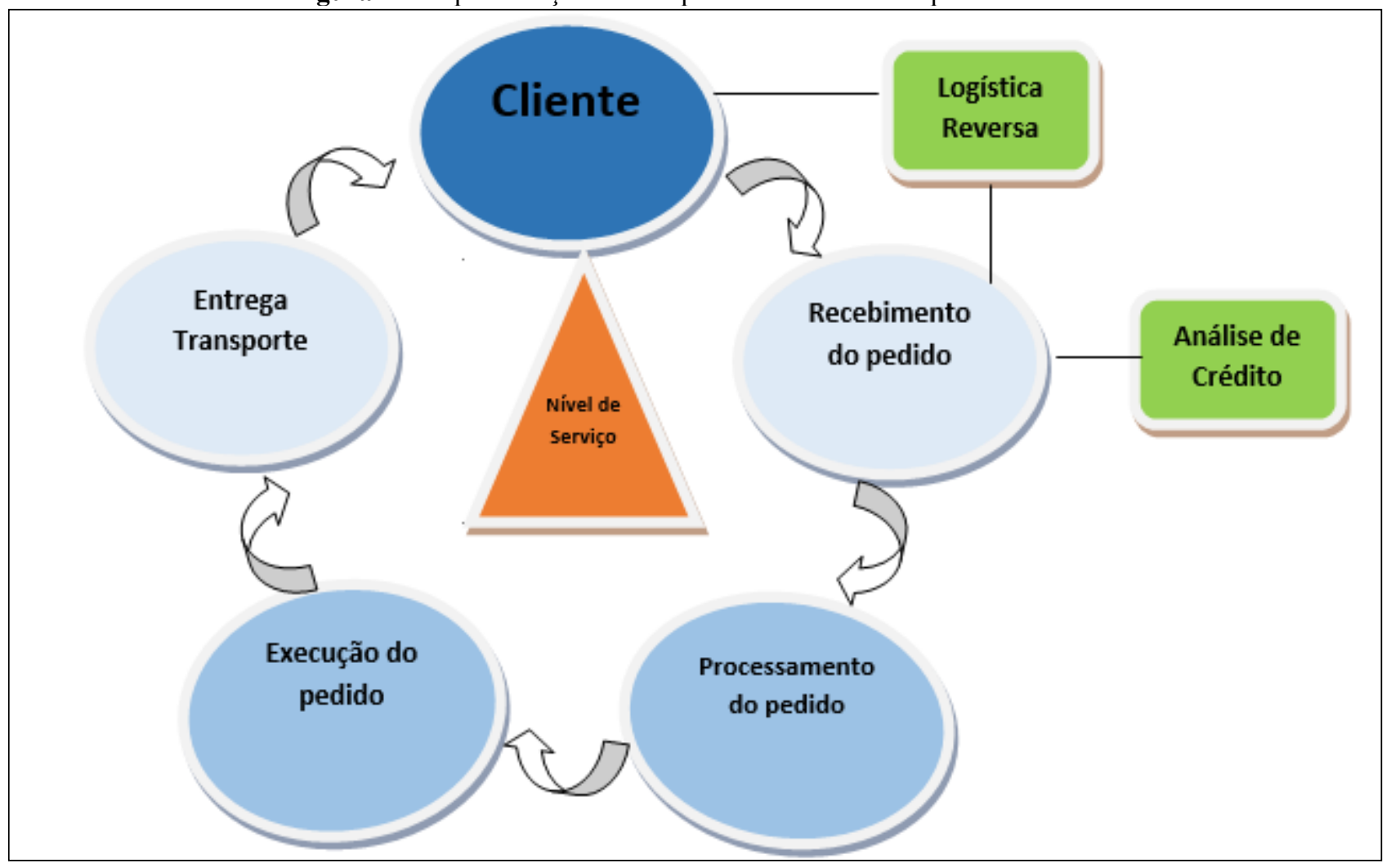

Fonte: Elaborado pela autora

A Figura 1 representa o ciclo de pedido, e destaca o cliente como ponto inicial, estes que estão cada vez mais compassivos ao tempo de espera, os grandes avanços tecnológicos proporcionaram troca de informação algumas vezes em tempo real, tornando os processos mais ágeis e seguros, proporcionando confiabilidade (SANTO, 2003). 


\subsection{Problemas na gestão do ciclo de pedido}

Embora a modernização dos processos do ciclo de pedido seja crescente, problemas podem surgir durante os subprocessos, Fleury (2003) destaca três problemas durante o ciclo mais frequentes.

1 - Percepções conflitantes: a percepção está relacionada em métricas distintas para os cliente e fornecedores, pois o "tempo de início do ciclo de pedido" inicia-se em tempos diferentes. O cliente mede o tempo de quando o pedido é formalizado até o recebimento do produto e para o fornecedor inicia-se quando o pedido é recebido e termina com a expedição e embarque dele. Fato que se não for observado e acompanhado pela empresa pode fazer com que o gestor logístico perca a rastreabilidade e mensuração do tempo estimado pelo cliente, enquanto o fornecedor pensa ter atendido as expectativas do cliente com excelência o cliente pode estar medindo como insatisfatório.

2 - Variabilidade de tempos: está diretamente relacionado a padronização, controles e acompanhamentos que garantem uma menor oscilação de tempo entre as atividades desenvolvidas. Os principais causadores de variação estão ligados as atividades de atrasos na transmissão de pedidos; aprovação de crédito; estabelecimento de prioridades; falta de estoque e atrasos no transporte.

3 - Flutuação da demanda: a oscilação na demanda são as incertezas do mercado e outros fenômenos que afetam o gerenciamento dos pedidos, tendo grande impacto no estoque e na disponibilidade dos produtos. Geralmente estão alinhados a promoções e campanhas de vendas.

Para Motta e Bianchini (2006), as reclamações dos clientes com relação a gerência de distribuição consideram como fatores de medição a pontualidade na entrega, índices de atendimentos aos pedidos, condições dos produtos e exatidão da documentação, estes que são considerados os mais importantes elementos logísticos no atendimento ao pedido. 


\section{MATERIAL E MÉTODOS}

\subsection{Metodologia}

O levantamento de dados bibliográficos foi realizado através de livros, revistas e periódicos das áreas de logística e da administração, além de pesquisar artigos científicos disponíveis na internet. Os dados foram fornecidos uma empreendedora autônoma que revende produtos cosméticos na região através de uma entrevista, porém a revendedora preferiu permanecer anônima.

O caráter da pesquisa é qualitativo, exploratório e o formato é de um estudo de caso real em uma empresa do ramo cosmético.

\subsection{Estudo de Caso}

O inglês Leonard Lauder, em 2001 criou um indicador chamado "Índice do Batom" (lipstickindex), onde a justificativa foi que o custo relativamente baixo do batom, é equivalente a elevação da auto-estima do seu usuário, ou seja, as estatísticas de vendas de cosméticos sobem em razão proporcional à queda do poder aquisitivo dos potenciais consumidores. Através da análise, esse índice demonstra que apesar das crises econômicas, as pessoas continuam investindo em cosméticos (ABEVD, 2018).

Segundo informações disponibilizadas no site da ABEVD (2018), o modelo de venda direta é, há muitos anos utilizado por diversas marcas no mercado mundial, entende-se desde grandes a pequenos negócios. A proposta era que através do modelo, vendessem seus produtos e serviços diretamente ao cliente final e sem a necessidade de um estabelecimento comercial fixo, com intuito de reduzir assim, os custos de distribuição. Os agentes responsáveis pela venda direta são denominados empreendedores independentes, comumente chamados de revendedores, consultores ou distribuidores.

Para dar base ao estudo de caso, foi realizada uma entrevista presencial com uma revendedora de cosméticos anônima de diversas grandes marcas, atuante na região de Bebedouro, SP. O roteiro levantou informações como: tempo de atuação, volume de vendas, tipo de planejamento de venda e entrega dos produtos, como funciona o relacionamento com o 


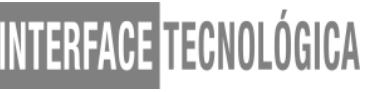

cliente, quais são as etapas da venda e controles utilizados de acordo como conhecimento da consultora.

Resumidamente, a consultora está atuante na revenda de produtos cosméticos de 3 marcas diferentes, com linhas de produtos de beleza, perfumaria, saúde e higiene. Tendo como público todos os gêneros, oferecendo opções de compra para todas as faixas etárias.

De acordo com os relatos, conseguiu-se mapear as atividades envolvidas nos processos de ciclo de pedido e suas etapas do início ao fim, ilustrado na Figura 3. O ciclo se inicia quando a revendedora vai até o cliente oferecer seus produtos, ou quando o cliente busca a revendedora para adquirir seus produtos. A próxima etapa se concretiza quando o cliente faz efetivamente o pedido com a revendedora, essa fase é crucial, pois nesse momento deve se haver atenção em coletar corretamente os dados do cliente e do pedido de forma a evitar erros que possam trazer insatisfação ao cliente, como anotar no pedido de venda o produto errado, pois nesse modelo, os produtos são tratados por códigos muitas vezes.

A terceira etapa consiste em verificar o estoque próprio ou levantar a necessidade de pedido com a distribuidora e também verificar a disponibilidade e prazos para o fornecimento, esta fase pode ser muito ampla e fugir do foco deste artigo, por isso será tratado de acordo com o ponto de vista de atender o cliente. Após essas atividades concluídas a revendedora prepara os produtos para serem entregues ao cliente final, pessoalmente ou através dos malotes ou serviços dos Correios, encerrando o de pedido.

\section{RESULTADOS}

A revisão bibliografia serviu de base para compreensão dos conceitos de nível de serviço e atendimento ao ciclo de pedido, como foco na satisfação do cliente. As informações que baseiam o estudo de caso dão norte ao resultado da pesquisa. Após a compreensão das atividades da revenda, destaca-se a importância de gerenciamento atividades envolvidas no processo. Como primeiro tratou de levantar as atividades envolvidas no ciclo de pedido, identificando onde ele se inicia e onde termina para uma revendedora e seu cliente final.

A Figura 2 ilustra de forma sistemática os elementos que compõem a distribuição de produtos no caso de revenda direta: 
Figura 2 - Principais agentes na distribuição de cosméticos através de venda direta.

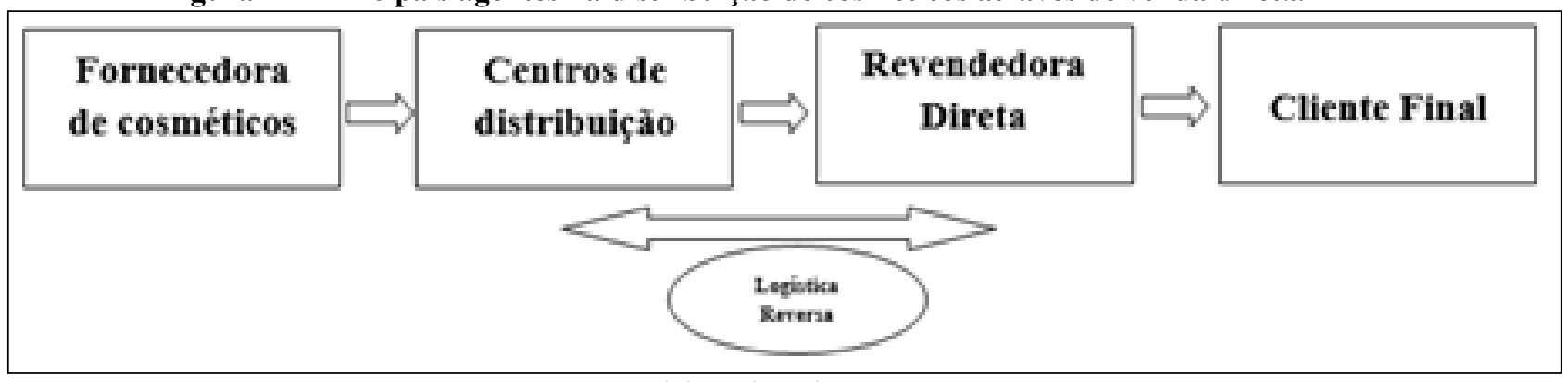

Fonte: Elaborado pela autora

Os agentes ilustrados na Figura 2 representam respectivos inícios de ciclo de pedido, da qual com sua perspectiva, neste caso o foco é o cliente final e a perspectiva analisada é da revenda direta. A próxima figura representa o ciclo de pedido da revenda direta:

Figura 3 - Ciclo de pedido revenda direta

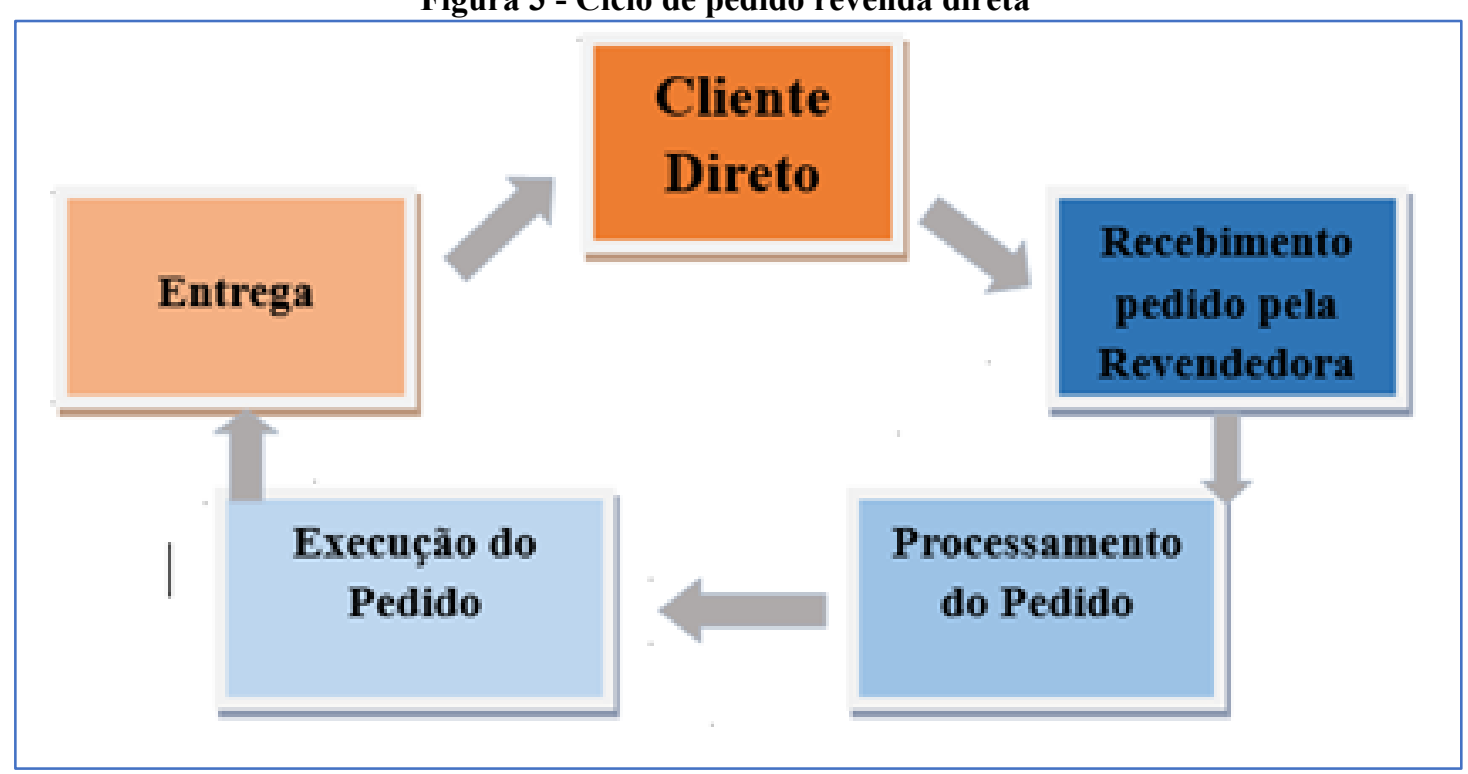

Fonte: Elaborado pela autora

No "recebimento do pedido" a revendedora precisa identificar todas as informações pertinentes à venda ao cliente direto solicitante, assim como realizar análise de crédito caso seja efetivada a compra a prazo. O ideal seria a utilização de uma ficha ou pedido de venda, documento que formaliza o pedido. Registrar em um documento de forma manual, em uma planilha do excel ou em um sistema, podem garantir confiabilidade das informações e minimizar erros e possíveis retrabalhos que deixariam o cliente insatisfeito e agregar custos extras ao processo de vendas. É recomendado também que a revendedora colete a assinatura do cliente, formalizando o pedido de venda (ABEVD, 2018). 


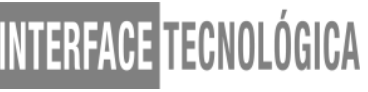

Durante a fase de "processamento do pedido" em que o ciclo é iniciado na prospecção da revendedora, deve ser analisada a disponibilidade do produto em seu estoque ou a necessidade de compra na distribuidora. Esta fase pode apresentar muitos problemas no tempo de atendimento do ciclo e impactar no nível de serviço ao cliente, pois nela está envolvido mais agente do processo, um exemplo é quando o produto não consta no estoque da revendedora e é necessário a interação com o distribuidor, este que tem seu próprio ciclo de pedido.

Uma boa opção para não comprometer o atendimento ao cliente, é levantar e manter históricos de vendas para darem base a estudos relacionados a viabilidade de estoque próprio. Após essa verificação da disponibilidade do produto, tem-se a separação e embalagem do pedido para a disponibilização para a entrega, seja por mãos próprias, terceirizadas ou por agentes dos correios. Os prazos de entrega impactam diretamente no ciclo, esse prazo deve ser estabelecido no início, no pedido de venda.

O cliente ciente do prazo cria expectativas com a entrega, e atender esse prazo com excelência trará satisfação ao cliente, que provavelmente fomentará o negócio com boas referências. E finalmente a entrega do produto ao cliente, encerra o atendimento ao ciclo de pedido.

Com tais informações detalhadas outro conceito pode ser aplicado, o de problemas no gerenciamento de ciclo de pedido. O problema de percepções conflitantes é facilmente identificado quanto a percepção de todos os agentes envolvidos no processo de distribuição para venda direta, assim como do cliente final. Neste caso, para o cliente final, o ciclo de pedido inicia-se quando a revendedora recebe o pedido e dá a confirmação de entrega, é muito importante levar tal fato em consideração, para não possibilitar que o cliente gere expectativas que não serão atendidas.

O problema relacionado a variabilidade de tempo está relacionado principalmente ao fato da "informalidade" e da simplicidade desse tipo de comercialização de produtos, na maior parte dos casos, não são usadas ferramentas para padronização e controle.

Com relação a oscilação da demanda, o estoque pode impactar em dois estágios do processo de atendimento ao pedido, primeiramente o estoque da revendedora e em segundo o estoque da distribuidora.

Diante das informações levantadas é possível a compreensão dos processos envolvidos no atendimento do pedido do cliente, considerando fatores de qualidade na prestação de serviço 
e efetividade na distribuição de produtos, possibilitando ao empreendedor (a) planejar estrategicamente seu negócio e conquistar mercado e satisfação de seus clientes.

\section{CONCLUSÃO}

Conclui-se que os objetivos foram alcançados, pois com os resultados obtidos, compreendeu-se a aplicabilidade dos conceitos de ciclo de pedido para o modelo de revenda direta da revendedora de cosméticos e, graças à teoria, pode-se sugerir a aplicação de conceitos de gerenciamento do nível de serviço e gestão logística em sua empresa.

Encontrou-se alguns problemas relacionados a variabilidade de tempo devido a "informalidade" em que essa empresa está submetida, devido a simplicidade desse tipo de comercialização de produtos e que, na maior parte dos casos, não são usadas ferramentas de gestão para a padronização dos seus processos e dos seus controles, que são feitos via excel.

Espera-se que o presente artigo estimule novos e mais estudos aprofundados relacionados ao nível de serviço, ao cliente e ao atendimento ao ciclo de pedido e inspire a formalização de revendas na região.

\section{REFERÊNCIAS}

ABEVID. O que é venda direta. 2018. Disponível em: <https://www.abevd.org.br/>. Acesso em: 10 fev. 2020.

BAE, $\mathrm{H}$. The influencing factors of logistics integration and customer service performance for value creation of port logistics firms. The Asian Journal of Shipping and Logistics, 2012.

BOWERSOX, D. J; CLOSS D. J; COOPER, M. B. Gestão Logística de Cadeia de Suprimentos. Porto Alegre: Bookman, 2006.

BOWERSOX, DONALD J. \& CLOSS DAVID J. Logistical Management: The intergraded Supply Chain Process. Nova York: McGraw - Hill, 1996

BOWERSOX, D. J.; CLOSS, D. J.; COOPER, M. B. Supply Chain Logistics Management. 2a Edição. ed. Nova York: McGraw - Hill, 2007.

CHRISTOPHER, M. O marketing da logística: otimizando processos para aproximar fornecedores e clientes. São Paulo: Futura, 1999. 
FLEURY, P. F.; WANKE, P.; FIGUEIREDO, K. F. Logística empresarial: a perspectiva brasileira. Coleção COPPEAD de Administração. São Paulo: Atlas, 2000.

FLEURY, A. Estrátegias Competitivas e Competências essenciais: Perspectivas Para a Intercionalização da Industria no Brasil. Revista Gestão e Produção, São Carlos, v.10, n. 2, p.129-144, ago., 2003.

LALONDE, B. J.; ZINSZER, P. H. Customer Service: Meaning and Measurement. Chicago National Council of Physical Distribution Management, 1976.

MAXIMIANO, A. C. A. Teoria Geral da Administração. 2.ed. São Paulo: Editora Atlas, 2008.

MOTTA G.A.; BIANCHINI, V.K. Análise do nível de serviço logístico em uma transportadora de cargas. 2010. Disponível em: $<$ http://www.abepro.org.br/biblioteca /enegep2010_tn_stp_113_741_17188.pdf >. Acesso em: 10 fev. 2020.

NOVAES, A. G. Logística e Gerenciamento da Cadeia de Distribuição. Rio de Janeiro: Elsevier, 2007.

SANTO, Angélica do Espírito. Considerações sobre a influência da análise das demonstrações no ciclo do pedido logístico: análise do caso Atofina Brasil. Revista de contabilidade e finanças [online]. 2003, p.101-113. Disponível em: $<$ http://www.scielo.br/scielo.php?script= sci_arttext\&pid=S1519-70772003000300008\&lng=en\&nrm=iso $>$. Acesso em: 10 fev. 2020. 